\title{
Termoscanner
}

\section{Raffaella Setti}

PUBBLICATO: 01 OCTOBER 2020

Termoscanner è una parola composta dal primo formante termo ('calore') e da scanner (dall'ingl. to scan 'esaminare, analizzare') creata per indicare sia persone che svolgono scansioni, sia strumenti che effettuano analisi secondo una sequenza prestabilita. Lo strumento è un misuratore della temperatura di una superficie o di un corpo (o di parti di esso), che funziona attraverso la rilevazione delle emissioni infrarosse, quindi senza necessità di contatto tra la parte analizzata e lo strumento stesso.

Bisogna subito precisare che non si tratta di uno strumento nuovo e neanche di una nuova tecnologia: la termografia infatti si basa sulla proprietà dei corpi di emettere una radiazione elettromagnetica strettamente collegata alla temperatura e strumenti a infrarossi per la misurazione di tale temperatura erano già diffusi in campo militare dall'inizio del XX secolo per intercettare oggetti o persone in mancanza di luce; sono inoltre ampiamente usati nel settore edilizio a partire dagli anni '6o del Novecento per valutare il livello di degrado degli edifici e, ancora più recentemente, hanno avuto un grande sviluppo in ambito medico per l'individuazione di inframmazioni tendinee o muscolari, per la diagnosi di tumori della pelle e, negli ultimi anni, anche per la localizzazione di quelli alla mammella.

Per applicare questa tecnica alla misurazione della temperatura corporea sono stati fondamentali gli studi di Francesco Pompei, fondatore della Exergen Corporation, che nel 1998 depositò il brevetto per il primo termometro dell'arteria temporale, inizialmente impiegato in pediatria, dove era forte l'esigenza di un'alternativa alla termometria dell'orecchio e a quella rettale; lo stesso Pompei nel 2004 pubblico l'articolo, Non-invasive temporal artery thermometry: Physics, physiology, and clinical accuracy) in cui si sottolineava l'utilità di tale sistema per la misurazione della febbre di grandi gruppi di persone, in quel momento particolarmente urgente per il contenimento dell'epidemia di Sars. E proprio in quell'occasione la Defense Science and Technology Agency (Dsta) di Singapore e la Singapore Technologies Electronics, nello stesso anno, misero a punto l'Infrared fever screening system (Ifss), il primo sistema a infrarossi utilizzato per lo screening di massa della temperatura corporea.

Con l'esplosione della pandemia di Covid-Ig questo strumento di prevenzione e contenimento si è diffuso capillarmente in luoghi normalmente frequentati dai cittadini; con lo strumento ha cominciato a circolare nella lingua comune anche il termine termoscanner, che possiamo considerare come neologismo dellitaliano non tanto per la novità dell'oggetto, quanto per l'improvviso e pervasivo ingresso del termine nel repertorio linguistico della maggior parte dei parlanti. Su Google libri la prima attestazione ditermoscanner si trova nel volume di Claudia Mongini e Giovanni Mongini, Storia del cinema di fantascienza. Dal 1995 al 1990 (Fanucci, I999, p. 86): "[...] rappresentare la creatura facendone vedere i tratti termici: con un termoscanner si è poi riusciti a riprodurre le gradazioni di colore del corpo umano, accentuandole o diminuendole con il solo ausilio del computer". Si tratta di una testimonianza che conferma l'esistenza della parola, anche se è evidente che l'ambito d'uso è ancora decisamente ristretto e specialistico. Dopo alcuni anni, la parola ricompare nel romanzo Per esclusione (di Andrea Novelli e Gianpaolo Zarini, Marsilio, 20o8, p. I7I): "C'erano anche due termoscanner a infrarossi, puntati sullinterrogato, con la funzione di rilevare ogni minima differenza di temperatura corporea", ma le occorrenze restano davvero limitate. 
Una ricognizione a ritroso su Google, con ricerca mirata di termoscanner alle sole pagine in italiano e relativa a intervalli decennali, restituisce qualche occorrenza (indicativamente un centinaio dal r99o al 2000 e poi circa 5.000 dal 2000 al 20I0), ma sono dati non affidabili in quanto la data è determinata dal lancio in rete dei siti, mentre i contenuti sono continuamente aggiornati e il termine termoscanner può ricorrere in testi pubblicati recentemente. Prendendo le singole annate 2018 e 2019 (ricerca effettuata il 20/08/2020), si conferma la presenza della parola già prima dell'esplosione della pandemia, ma con numeri decisamente inferiori a quelli attuali: 55.500 occorrenze per l'intero 20 o 8 e 2r.90o per il 2019 , mentre in questi primi otto mesi del 2020 le occorrenze sono arrivate a 78.600 superando anche quelle di termometro digitale che nello stesso intervallo di tempo del 2020 conta 70.500 occorrenze (73.500 nel 2018 e 8I.900 nel 2019).

Da strumento conosciuto e utilizzato solo da esperti e professionisti di alcuni particolari settori, il termoscanner è diventato un apparecchio quotidiano che le persone comuni hanno potuto vedere, sperimentare direttamente e di cui molti hanno dovuto studiare e analizzare prestazioni, funzionamento e costi. Con l'oggetto e l'obbligo del suo utilizzo in luoghi pubblici molto frequentati, anche il nome ha cominciato a circolare fino a diventare un termine sulla bocca di tutti. Come ha notato Sergio Lubello, "sono soprattutto tecnicismi della medicina i 'nuovi' ingressi nel lessico quotidiano, sprovvisti di aura e di mistero: molti sono diventati termini prêt-à-porter [...] altre parole disegnano nuove abitudini di controllo igienico-sanitario: il termoscanner e il (gel) disinfettante o igienizzante" (Nuovo lessico famigliare: le 100 parole della pandemia, treccani.it).

In questi ultimi mesi abbiamo avuto tutti l'esperienza pero di ritrovarci di fronte (nel vero senso della parola!) a strumenti differenti: da piccoli termometri a forma di pistola mirati sulle nostre fronti a termocamere collegate a computer portatili (simili a quelli dei metal detector) in cui compare l'immagine della scansione termica del nostro corpo, fino ai totem a cui bisogna accostarsi avvicinando il viso a uno schermo. Le differenze tra questi strumenti riguardano la forma, il costo e naturalmente la precisione e quindi l'affidabilità (dipendente anche dal corretto uso), ma il principio di funzionamento è lo stesso:

Le "pistole" per misurare la temperatura usate all'ingresso di molti locali hanno dei limiti e devono essere usate con attenzione per essere utili. [...] All'interno di tutti i termoscanner è presente una termocamera, cioè una videocamera sensibile alla radiazione infrarossa e che permette di realizzare "mappe di temperatura" di ciò che si sta inquadrando, o, nei termoscanner più piccoli, un sensore che fa la stessa cosa. (Perché i termoscanner sono imprecisi, ilpost.it, 30/05/2020)

Inizialmente una certa varietà, oltre che nella forma dell'oggetto, è stata messa in evidenza anche nelle denominazioni:

Vengono chiamati in mille modi: termoscanner, termometri laser, termometri a distanza, termometri ad infrarossi. Tanti nomi, per indicare uno degli strumenti più discussi degli ultimi giorni, diventati una delle armi principali per il controllo della temperatura, soprattutto negli aeroporti, dopo la grande diffusione del Covid-rg. (Dario Caliendo, Come funziona la pistola per misurare la temperatura, fanpage.it, $24 / 2 / 2020)$

Riguardo a questo, in base a una ricognizione in rete (Google al $17 / 08 / 2020$, pagine in italiano), possiamo dichiarare come nettamente prevalente la forma termoscanner ( 984.000 occorrenze, oltre a 82.900 occorrenze complessive per le varianti termo scanner e termo-scanner e 23.000 per la grafia con $h$ etimologica thermoscanner) a fronte delle 56.800 per termometro a infrarossi, delle 24.000 per termometro a distanza e delle 21.0oo per termometro laser. Compare, ma molto più sporadicamente, anche la denominazione di termorilevatore a distanza (9 occorrenze all'r/og/2020) o di temperatura (37 
occ. una soltanto per termorilevatore di febbre).

La sintesi in un'unica parola e la conoscenza diffusa dei formanti (termo è presente in moltissimi composti dell'italiano, si pensi solo a termometro, termoconvettore, termodinamica; lo scanner è ormai uno strumento largamente impiegato) hanno certamente contribuito in modo determinante all'affermazione di termoscanner, anche se nella selezione delle forme da parte dei parlanti sembra funzionare la distinzione tra termoscanner, apparecchiatura più sofisticata e adatta allo screening di massa, rispetto a termometro (a distanza, a infrarossi, laser) per riferirsi allo strumento "a pistola" che consente la misurazione della temperatura sempre a distanza, ma con minor precisione e velocità (quindi adatto a luoghi in cui il flusso di persone è meno intenso).

La prima attestazione rintracciata di termoscanner nella recente accezione è contenuta in un comunicato dell'agenzia AGI del 23 gennaio 2020, in cui si riportano anche le spiegazioni fornite dal direttore sanitario degli aeroporti di Roma, Carlo Racani, sulla procedura e sul funzionamento delle macchine:

Controlli sanitari con un termoscanner per il controllo della temperatura corporea sono stati attivati questa mattina all'alba all'aeroporto di Fiumicino per gli oltre 200 viaggiatori provenienti da Wuhan, la città della Cina epicentro del Coronavirus. Per la prima volta è stato attivato il percorso sanitario disposto dall'Usmaf del ministero della Salute. [...] Il canale sanitario è una struttura apparentemente avveniristica - spiega Racani - ma la procedura è molto semplice. Le persone passano sotto le telecamere termiche. Se il colore della pelle cambia, il passeggero viene enucleato, portato fuori dal gruppo e da lì iniziano i controlli specifici per poter vedere di che cosa si tratta. (Virus Cina: termoscanner a Fiumicino per 200 arrivati da Wuhan, agi.it, $23 / \mathrm{I} / 2 \mathrm{O} 20$ )

Il termine non compare invece nei primi documenti ufficiali del Governo e del Ministero della salute (quelli emanati tra la fine di gennaio e i primi di febbraio 2020) e non è stato citato esplicitamente neanche nella Conferenza stampa congiunta (Giuseppe Conte e Roberto Speranza) del 30/or/2020 in cui il Presidente del Consiglio Conte ha parlato di "principio di precauzione", espressione che, nel resoconto giornalistico, è stata sciolta, con riferimento chiaro alla misurazione della temperatura corporea, senza però citare lo strumento (o gli strumenti) impiegato a tale scopo:

Il Presidente ha ricordato che, sin dalle prime fasi dell'epidemia, ispirandosi al principio di precauzione, in Italia sono stati implementati controlli aeroportuali per i cittadini provenienti dalla zona sede del focolaio epidemico e attuato accurate misure di controllo - misurazione della temperatura corporea, identificazione ed isolamento dei malati, procedure per il rintraccio e la quarantena dei contatti stretti che, unitamente ad un efficiente sistema di sorveglianza epidemiologico e microbiologico, garantiscono il rapido contenimento di eventuali casi. (Conferenza Stampa del Presidente del Consiglio Giuseppe Conte e del Ministro della Salute Roberto Speranza, 30/or/2020)

L'introduzione dei termoscanner negli aeroporti (dal 23 gennaio a Fiumicino) e nei porti produce una prima ondata di utilizzo della parola nei mezzi di comunicazione di massa e anche nel linguaggio dei politici:

Il Comitato Operativo è tornato a riunirsi al fine di valutare tutti gli scenari operativi legati all'attuale livello di diffusione del virus e predisporre le misure da adottare nel brevissimo periodo, in particolare per quanto concerne il traffico aereo e quello marittimo.

Tra queste ultime rientra l'aumento dei controlli sanitari anche nei porti, con l'estensione di una procedura finora prevista soltanto per le imbarcazioni non Ue, la "libera pratica sanitaria", a tutte le imbarcazioni mercantili e da crociera in transito, e l'impiego di termoscanner negli aeroporti, per monitorare le condizioni di salute dei passeggeri dei voli che provengono dalla Cina attraverso scali 
intermedi. (Coronavirus, le misure adottate dal Governo, sul sito governo.it, I/o2/2020)

Lo stesso Giuseppe Conte, in un'intervista del 26/02/2020, utilizza il termine per evidenziare l'efficienza e la velocità dello strumento:

Dopo avere disposto il divieto dei voli diretti abbiamo immediatamente predisposto l'adozione di alcune misure per tutti i voli provenienti dall'estero. Con i termoscanner abbiamo controllato tutti i passeggeri che sono sbarcati nei nostri aeroporti, soltanto a Fiumicino circa 50.000 passeggeri al giorno. (Monica Guerzoni, Intervista a Giuseppe Conte, "Corriere della Sera", 26/o2/2020)

$\mathrm{Dal}$ 22/02/2020 viene prescritto anche agli istituti penitenziari il rispetto delle indicazioni del Ministero della salute con la predisposizione di controlli all'ingresso dei nuovi detenuti, anche tramite termoscanner, come riferito alcuni mesi dopo dal Ministro della giustizia Bonafede:

Per evitare ciò il 22 febbraio, il capo Dipartimento, l'allora capo Dipartimento, dottor Basentini, prescriveva il rispetto da parte degli istituti penitenziari e del personale delle indicazioni provenienti dal Ministero della salute, invitando allo stesso tempo ogni direzione a coordinarsi con le autorità sanitarie locali. [...]

Predisposizione di controlli in ingresso, anche tramite termoscanner, dei detenuti cosiddetti nuovi giunti, nonché del personale dell'amministrazione penitenziaria. (20/05/2020, Sito del Senato della Repubblica: http://www.senato.it/service/PDF/PDFServer/BGT/I152667.pdf).

Il periodo in cui però il termine ha visto la sua massima diffusione è stato senza dubbio a conclusione del confinamento, nel corso del mese di maggio, quando si sono fissate progressivamente tutte le misure di prevenzione che i locali pubblici, in particolare quelli con flussi consistenti di persone, avrebbero dovuto adottare per poter riaprire in sicurezza. Almeno i maggiori quotidiani ci mostrano questa tendenza; infatti negli archivi della "Repubblica" del "Corriere della Sera" e della "Stampa" in rete si rileva nel mese di maggio un innalzamento, rispetto ai mesi precedenti, delle occorrenze di termoscanner con una buona tenuta nella prima metà del mese successivo: 40 occorrenze sul "Corriere della Sera" (nessuna a gennaio, 7 a febbraio, 8 a marzo e iz nella prima metà di giugno); I 88 occorrenze sulla "Repubblica" (nessuna a gennaio, 24 a febbraio, 53 a marzo, 70 ad aprile e 90 nella prima metà di giugno); ig sulla "Stampa" (nessuna a gennaio, 5 a febbraio, II ad aprile e soltanto 2 nella prima metà di giugno). Una conferma in questa direzione è la curva che ci restituisce Google Trends con un picco di ricerche di termoscanner (finalizzate all'acquisto) proprio nella settimana tra il 3 e il 9 maggio. 


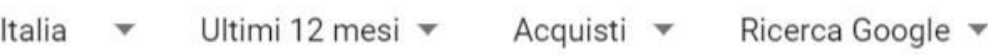

Interesse nel tempo
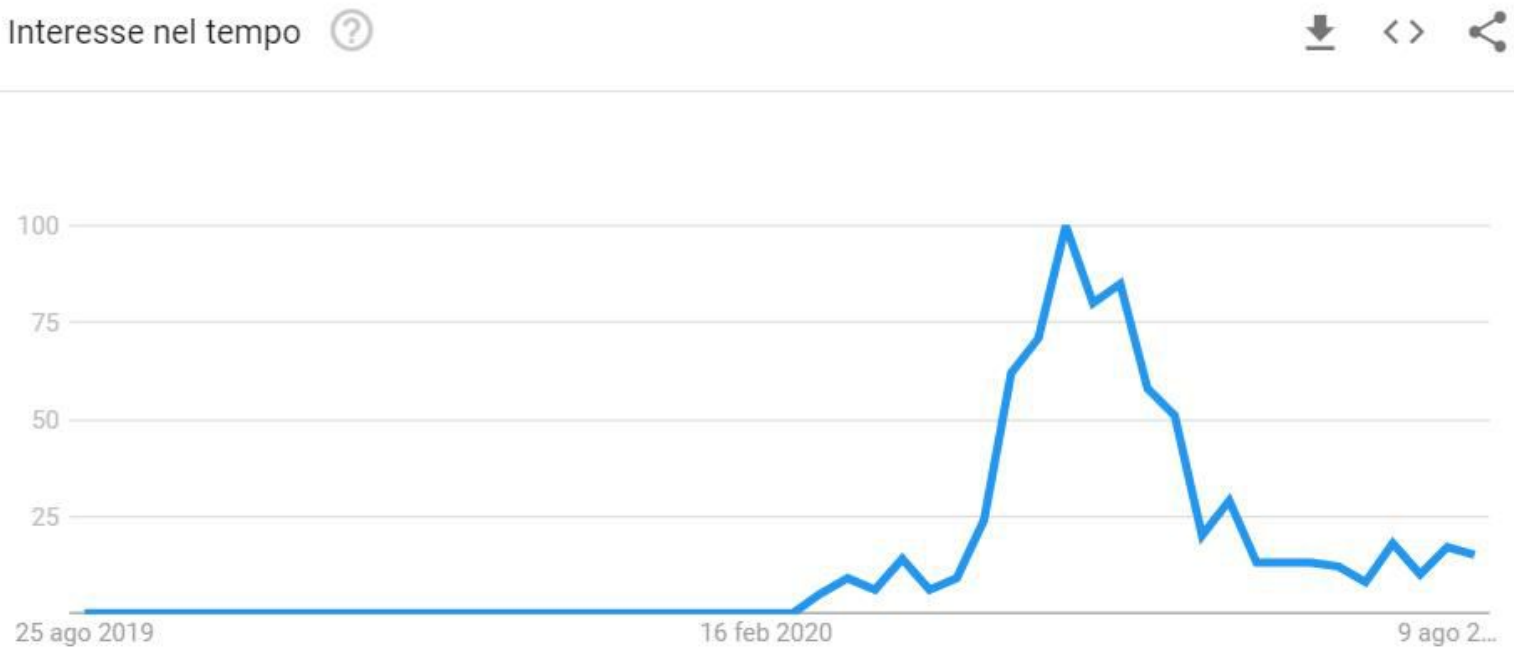

Leggermente diverso invece l'andamento delle occorrenze in rete, che mostra una sistematica anticipazione dei picchi di attestazione della parola, con il massimo delle occorrenze, 35.700, nel mese di aprile (Google, pagine in italiano periodo I-30 aprile), una flessione nel mese di maggio con 22.90o occorrenze e una ripresa a giugno con 35.60o. Possiamo forse attribuire la ragione di questa diversa frequenza nel tempo alla capacità del mercato di prevedere l'impennata delle vendite di questi strumenti e la conseguente campagna informativa e promozionale in vista della riapertura. La misura adottata fin da gennaio negli aeroporti lasciava presupporre che, al momento della ripartenza, l'obbligo della misurazione della temperatura corporea sarebbe stato esteso ad altri luoghi di grande transito, come poi infatti è avvenuto: le strutture sanitarie, le stazioni ferroviarie, i luoghi di culto, le grandi aziende, le istituzioni pubbliche, gli impianti sportivi, i centri commerciali, i musei, teatri e cinema (anche all'aperto), le biblioteche, oltre a molti esercenti, per poter riprendere le attività, hanno dovuto dotarsi di questo apparecchio. La seconda impennata delle occorrenze nel mese di giugno si spiega poi con l'adeguamento alle misure di sicurezza anche da parte delle strutture ricettive in vista della stagione estiva; c'è stata di conseguenza una grande richiesta di informazioni e unofferta esorbitante, soprattutto in rete (ormai il canale largamente prioritario per l'approvvigionamento di beni di consumo di ogni tipo), di termoscanner, dai più sofisticati capaci di operare su flussi continui di persone, fino ai più economici e maneggevoli termometri "a pistola": se n’è dunque parlato e scritto molto, si è polemizzato sull'affidabilità dei diversi tipi di strumento e sull'impennata dei prezzi, insieme alla quale si è avuta anche l'impennata della frequenza d'uso della parola.

Coronavirus a Brescia: Fase 2, ok ai funerali anche senza termoscanner, ma la Diocesi si attrezza per fornirli (Lilina Golia, "Corriere della Sera", 03/05/2020)

Coronavirus a Bergamo: è caccia ai termoscanner: obbligatori e a caro prezzo. Aziende e negozi devono misurare la temperatura ai dipendenti. [...] Per i termoscanner dobbiamo constatare che i prezzi sono raddoppiati. [...] Si è scatenata così una ricerca agli apparecchi a infrarossi per misurare la febbre a distanza i cui prezzi si sono impennati (Pietro Tosca, Coronavirus a Bergamo: è caccia ai termoscanner: obbligatori e a caro prezzo, "Corriere della Sera", I6/05/2020)

Lazio, pienone sulle spiagge del litorale tra termoscanner e app" (Valeria Costantini, "Corriere della Sera", or/o6/2020) 
Si moltiplicano poi gli aggiornamenti e gli avvisi nei siti istituzionali per informare i cittadini e i dipendenti pubblici dell'introduzione di tale misura; molte università, ad esempio, emanano informative sull'uso dei termoscanner per informare i dipendenti sul trattamento dei dati sensibili:

Informativa sul trattamento dei dati personali. Rilevazione della temperatura corporea attraverso termoscanner. Con questo documento La informiamo che l'Università degli Studi di Catania, in questa fase di criticità sanitaria determinata dal COVID-rg prevede, in via temporanea e sperimentale, l'attuazione di misure di controllo e accertamento volti a contenere e ridurre e il rischio di contagio (dal sito dell'Università di Catania, II/05/2020).

Lobbligo di sottoporsi a controllo della temperatura corporea mediante termoscanner installati in prossimità dell'accesso alla sede prima dell'ingresso al luogo di lavoro. Ove nella sede non fosse presente il termoscanner, il personale si recherà nella sede più vicina dove la strumentazione è presente, al fine della rilevazione della temperatura, prima di accedere al posto di lavoro (dal sito dell'Università Roma Tre, 22/05/2020).

La rilevazione della temperatura corporea viene effettuata in tempo reale a tutti i soggetti (dipendenti, studenti, assegnisti, borsisti, contrattisti, fornitori, visitatori etc) all'atto di accedere nella sede dell'Università oggetto di monitoraggio senza registrazione della temperatura stessa. Il termoscanner non registra né conserva dati personali, neanche dei soggetti la cui temperatura corporea riscontrata sia superiore a $37.5 \mathrm{C}^{\circ}$ (dal sito dell'Università politecnica delle Marche, $\left.\mathrm{Io} / \mathrm{o6} / 2020\right)$.

Sul sito dell'Università di Firenze viene pubblicato tra le notizie (intorno al 20 maggio, ma la notizia non è più visibile) un avviso in cui si utilizza la grafia separata ("CORONAVIRUS: variazione oraria del servizio di rilevazione della temperatura prima dell'ingresso alle sedi di lavoro. Da mercoledi 27 maggio i servizi di termo scanner osserveranno i seguenti orari"), mentre la successiva informativa ufficiale del 26/06/2020 cita i "Termometri digitali per la misurazione delle febbre a distanza". Un recentissimo comunicato dell'Ateneo fiorentino fa riferimento a "sistemi di rilevazione di temperatura", espressione sintetizzata nella comunicazione interna in termorilevatori. Una delle tante possibili conferme alla compresenza di grafie e denominazioni diverse con la scelta, almeno nel documento ufficiale, della forma tutta italiana anche se meno economica dal punto di vista linguistico.

Diversa appare la situazione negli altri paesi europei, dove termoscanner non sembra essere una parola corrente: non è certamente frequente in inglese, nemmeno nella forma thermoscanner, non solo nei dizionari (non è registrato neanche nell'Urban Dictionary), ma neanche sui principali giornali (sul "New York Times" si rintraccia temperature check); in francese non compare né su "Le Monde" né su "Libération"; in spagnolo non sembra usato (raro in Spagna anche l'utilizzo dello strumento), ed eventualmente la denominazione è quella di cámara térmica (presente in rare occorrenze sul quotidiano "El Pais"); in tedesco, sulla base di testimonianze dirette di colleghi linguisti e medici, sembra non sia diffusa una parola diversa rispetto a quella per indicare il termometro tradizionale, anche se proprio dalla Germania ci arriva la denominazione ThermoScan ${ }^{\circledR}$, marchio registrato da almeno 25 anni dalla Braun per indicare il termometro auricolare per neonati e bambini; visto che prevede necessariamente il contatto fisico, tale termometro non è certamente adatto a screening di massa, ma sul piano formale il suo nome ricorda quello del termoscanner, tanto da far pensare a un rapporto diretto tra le due denominazioni.

Il termine, almeno per adesso, non è contemplato nemmeno in IATE, la banca dati terminologica dell'Unione europea (https://iate.europa.eu/), a conferma della sua circolazione quasi esclusivamente italiana che quindi non pone problemi di uniformità con le eventuali forme delle altre lingue europee.

In conclusione, termoscanner non appare come un vero e proprio nuovo conio dellitaliano, ma un 
termine che, a più riprese, negli ultimi mesi ha avuto un rilancio davvero consistente e pervasivo che ne ha determinato l'uscita da ambiti d'uso esclusivamente specialistici e la diffusione nella lingua comune. Possiamo prevedere che sarà uno strumento con cui avremo a che fare in modo costante anche in futuro e che quindi anche la circolazione della parola proseguirà. Nelle prossime nuove edizioni dei vocabolari scopriremo se i lessicografi promuoveranno termoscanner a parola da inserire nel lessico comune o se invece continueranno a considerarlo un termine specialistico.

\section{Cita come:}

Raffaella Setti, Termoscanner , "Italiano digitale", 2020, XV, 2020/4 (ottobre-dicembre)

DOI: $10.35948 / 2532-9006 / 2020.4402$

Copyright 2020 Accademia della Crusca

Pubblicato con licenza creative commons CC BY-NC-ND 